\title{
Applications of combined spectral lifetime microscopy for biology
}

\section{Long Yan, Curtis T. Rueden, John G. White, and Kevin W. Eliceiri}

Laboratory for Optical and Computational Instrumentation

University of Wisconsin-Madison, Madison, WI, USA

Live cell imaging has been greatly advanced by the recent development of new fluorescence microscopy-based methods such as multiphoton laser-scanning microscopy, which can noninvasively image deep into live specimens and generate images of extrinsic and intrinsic signals. Of recent interest has been the development of techniques that can harness properties of fluorescence, other than intensity, such as the emission spectrum and excited state lifetime of a fluorophore. Spectra can be used to discriminate between fluorophores, and lifetime can be used to report on the microenvironment of fluorophores. We describe a novel technique-combined spectral and lifetime imaging - which combines the benefits of multiphoton microscopy, spectral discrimination, and lifetime analysis and allows for the simultaneous collection of all three dimensions of data along with spatial and temporal information.

\section{Introduction}

All living organisms are cells or ensembles of cells. This insight $(1,2)$, arguably the most significant in all of biology, was made possible by the development of the light microscope (3). One hundred and sixty-seven years later, the microscope is still at the forefront of biomedical research into the structure and dynamics of subcellular components in living tissue. The remarkable developments in molecular biology and genomics during the past few years have provided DNA sequences of many organisms, from which the complete complement of proteins making up these organisms can be deduced. However, knowledge of a comprehensive "parts list" of an organism does little in and of itself to explain the workings of the dynamic machinery that underlies the ability of a cell to divide or differentiate. It is light microscopy that is providing the key insights into subcellular dynamics, a task being greatly facilitated by technical developments in optical probes and instrumentation. Chimeric fluorescent protein reporters (4-10) allow virtually any protein to be labeled and thereby visualized in a cell, tissue, or organism. Fluorescent molecules have been engineered to reveal key intracellular physiological parameters, such as free calcium levels (11-13). Along with these developments in fluorescence probe technology, new optical techniques, such as fluorescence resonance energy transfer (FRET) (14-17), multiphoton laser-scanning microscopy (MPLSM) (18-21), second harmonic generation (SHG) imaging (22-25), and fluorescence lifetime imaging (FLIM) (26-30), are revealing how individual cellular components are assembled into cytoplasmic machinery and how this machinery functions. In this review, we will describe a relatively new technique, spectral lifetime imaging microscopy (SLIM), that simultaneously measures fluorescence lifetime and spectra for intrinsic and extrinsic fluorophores. This technique can utilize the benefits of
MPSLM and has great promise in revealing more information about intrinsic and extrinsic fluorophores and their interactions with each other and with their microenvironment.

MPLSM has proven to be a powerful tool for imaging cancer and cell biology phenomena $(19,20,31-34)$ by allowing scientists to image noninvasively deep into biological tissue and collect four-dimensional data [three-dimensional (3-D) spatial dimensions plus time] of fluorescently labeled proteins. Recently, there has been a growing awareness that there are properties of fluorescence other than intensity, such as lifetime (how long a photon stays in the excited state) and spectra. These properties can be used to separate out fluorophores of interest (via spectra) and to reveal critical information about the microenvironment (via lifetime), as well as about the abundance and bound state of key endogenous fluorophores, such as nicotinamide adenine dinucleotide (NADH) $(29,35)$ and flavin adenine dinucleotide (FAD). Until recently, all these dimensions of data have been collected separately, but now our group and others have developed instrumentation for simultaneous collection of intensity, space, time, spectra, and lifetime (36-38). This combined information can allow for the determination of the "fingerprint" of an intrinsic or extrinsic fluorophore in space and time, which allows investigators to track fluorophores accurately and determine their role in key processes.

\section{Fluorescence Microscopy}

Fluorescence microscopy has become the foremost tool in the study of the structure and dynamics of cellular machinery in living cells and tissue because of the high signalto-background ratio that may be obtained by observing fluorescent objects against a black background, together with the ability to spectrally discriminate between mul- 


\section{Tech Insight}

tiple fluorophores, These fluorophores can be endogenous metabolites (e.g., reduced NADH), genetically engineered proteins [e.g., green fluorescent protein (GFP)::tubulin], or exogenous probes [e.g., fluorescence microscopy (FM) series lipid probes or the $\mathrm{Ca}^{2+}$ indicator Fura]. Each fluorophore has a characteristic emission spectrum that may be used for identification purposes. In addition, fluorophores have characteristic excited state lifetimes, which can be used also to aid identification (28). Excited state lifetimes - and, to a lesser extent, fluorescence spectra-can be modified by the microenvironment of the fluorophore and therefore can be used to report on it. By utilizing photon-counting detectors, the fluorescence lifetime of individual fluorophores, such as the bound and free forms of $\mathrm{NADH}$, can be tracked and recorded for metabolic mapping in breast cancer (29).

\section{Optical Sectioning Techniques}

Optical sectioning techniques such as confocal laser-scanning microscopy $(39,40)$ or MPLSM $(19,21)$ have been developed that provide images from within a solid specimen that are free of interference from out-of-focus signals arising from fluorescent structures above and below the plane of focus. Stacks of optical sections can be collected enabling the 3-D structures of living specimens to be visualized over time $(21,41,42)$. MPLSM is based on the nonlinear interactions between light and excitable molecules (21). At very high photon densities, two or more photons may be simultaneously absorbed by a fluorescent molecule causing it to fluoresce, provided the sum of the individual photon energies is equivalent to the energy required for a single photon to induce fluorescence. In the case of twophoton imaging, the excitation wavelength is set to about twice that of the absorption peak of the fluorophore being observed. Normally, this wavelength would not produce any appreciable excitation. However, if a high-power, ultrashort pulse laser is used, it is possible to achieve instantaneous photon densities that are sufficient to give rise to a significant yield of two-photon events in the focal volume (or focus scan area) of an objective lens, while maintaining a mean power level that will not damage the specimen. An additional advantage of MPLSM for in vivo studies is that phototoxic effects are minimized because fluorophore excitation is confined to the plane of the optical section being observed and longer wavelengths are utilized $(21,43)$. Multiphoton imaging is the ideal instrumentation base for a SLIM system, as it provides the fluorescence excitation needed for spectral and lifetime collection, while retaining the viability and deep section benefits of MPLSM.

\section{Fluorescence Lifetime Imaging}

FLIM is a recently developed imaging mode where the excited state lifetimes of the fluorophores within a speci- men are revealed (usually in false color) $(28-31,44)$. The lifetime of the excited state, which gives rise to the emitted fluorescence photons, provides another dimension of information that is often independent of color. This extra dimension of data may be particularly useful in identifying fluorophores with significantly overlapping spectral properties. The excited-state lifetime is diagnostic of the fluorophore and also of its microenvironment $(26,45)$. Factors such as ionic strength, hydrophobicity, oxygen concentration, binding to macromolecules, and the proximity of molecules that can deplete the excited state by resonance energy transfer can all modify the lifetime of a fluorophore. Measurements of lifetimes can therefore be used as indicators of these parameters. Fluorescence lifetime measurements are generally absolute, being independent of the concentration of the fluorophore. Lifetimes may be measured in either the frequency or time domains. We favor time domain measurements, because they use photon-counting techniques that minimize the effects of noise sources such multiplier gain noise in photodetectors and shot noise in analog amplifiers.

\section{Spectral/Lifetime Multiphoton Imaging System}

We have implemented a multiphoton imaging system that uses an innovative combined spectra/lifetime detector optimized for in vivo studies. When a fluorescence photon is emitted from a molecule within a living cell, it carries a signature that can potentially identify the molecule and provide information on the microenvironment in which it resides, thereby providing insights into the physiology of the cell. To unambiguously identify fluorescent probes and

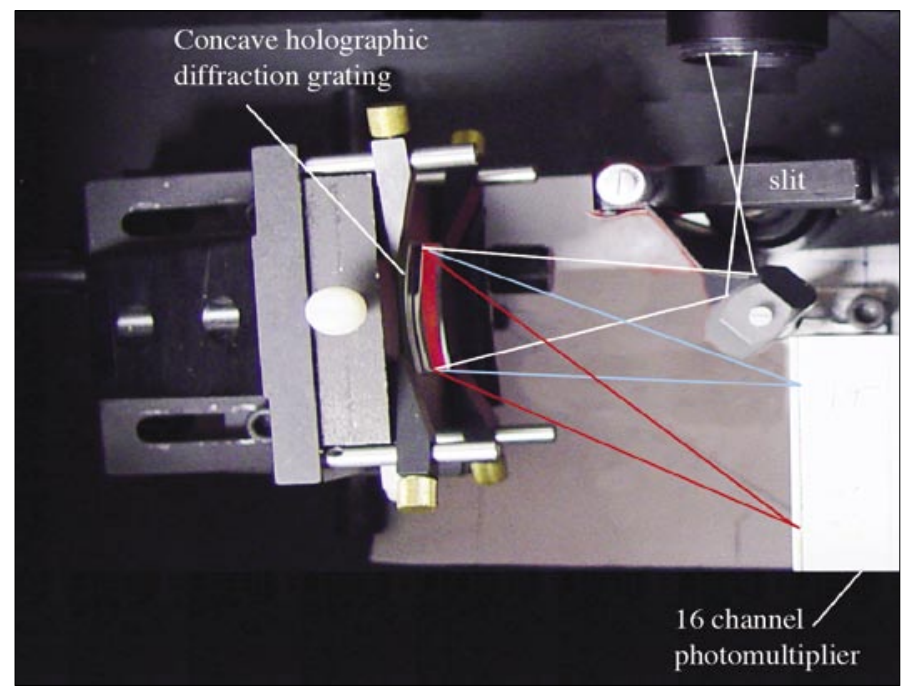

Figure 1. The spectrometer uses a concave, aberration-corrected holographic diffraction grating that provides a flat field and requires no auxiliary focusing optics. It is directly attached to the side-port of a Nikon Eclipse microscope via a condenser lens (not shown) that images the back aperture of the objective onto an adjustable slit. A mirror directs the beam onto the diffraction grating, which re-images the slit on the front face of the photomultiplier. 


\section{Tech Insight}
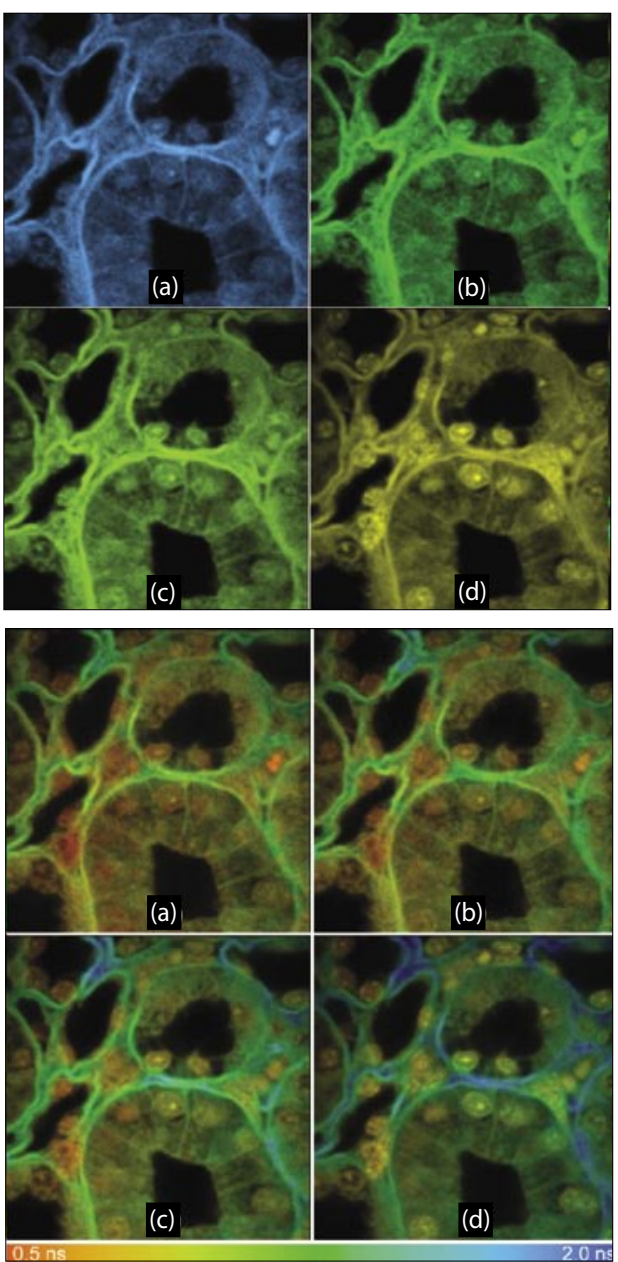

Figure 2. Multiphoton laser-scanning microscopy (MPLSM) spectral/lifetime images of a transverse section though the medulla of a Cynomolgus monkey kidney stained with methyl green. Top panel shows multiphoton intensity images acquired at the (a) $480 \mathrm{~nm}$, (b) $510 \mathrm{~nm}$, (c) $550 \mathrm{~nm}$, and (d) $580 \mathrm{~nm}$ wavelength components of the emission spectrum, and bottom panel shows pseudocolor fluorescence lifetime images also acquired at the (a) $480 \mathrm{~nm}$, (b) $510 \mathrm{~nm}$, (c) $550 \mathrm{~nm}$, and (d) $580 \mathrm{~nm}$ spectral intervals ( $\pm 10 \mathrm{~nm}$ ).

monitor their physiological environment within living specimens by their fluorescent signatures, one must exploit as much of this information as possible. Our combined two-photon spectral and lifetime microscope can collect fluorescence lifetime images from 16 individual wavelength components of the emission spectrum with $10-\mathrm{nm}$ resolution on a pixel-by-pixel basis.

Our current spectrometer uses a blazed, aberration corrected, holographic diffraction grating (Shimadzu 20046). These devices provide a flat field and so are ideally matched to a linear detector, requiring no additional focusing optics. Blazing has only recently been available on holographic gratings and has enabled efficiencies of $>50 \%$ to be achieved around the blaze wavelength. The grating that we use has a wide $\mathrm{f} 2$ aperture, yet is small with a short $(86 \mathrm{~mm}$ ) focal length allowing a compact spectrometer to be designed that can be mounted directly on the side-port of a Nikon Eclipse TE2000-U microscope (Nikon, Melville, NY, USA) (Figure 1). Although this configuration loses some of the convenience of optical fiber coupling, we gain sensitivity as we can use a slit rather than a circular aperture for the spectrometer and also do not suffer any fiber coupling losses. The slit is adjustable so that trade-offs between spectral resolution and sensitivity can be made.

\section{Examples of Biological Studies that Can Utilize SLIM}

We have been exploring the possible application of spectral/lifetime imaging as a diagnostic tool for histologists to differentiate normal and malignant structures. In a pilot project, we have shown that FLIM can be used on classically stained histology slides to help differentiate between structures based on changes in lifetime (30). Initial studies have indicated that the extra dimensions of information provided by spectral/lifetime imaging could facilitate diagnostic judgments (Figure 2). We are now applying this work to standard hematoxylin and eosin (H\&E) histopathology studies to investigate whether the extra information can facilitate the identification of diseased tissue from normal in standard path-lab specimens.

We have begun pilot studies in utilizing SLIM to study metabolism in breast cancer. Previous work demonstrated that FLIM can be utilized to detect the free and bound forms of NADH in human breast cell lines (29). We have now begun to utilize SLIM to look at NADH in both normal and malignant cell lines (Figure 3). The advantage of SLIM is that not only does it allow for detection of the free and bound forms of NADH via lifetime, but it also helps distinguish $\mathrm{NADH}$ from the other intrinsic fluorophores (such as FAD) via spectra.
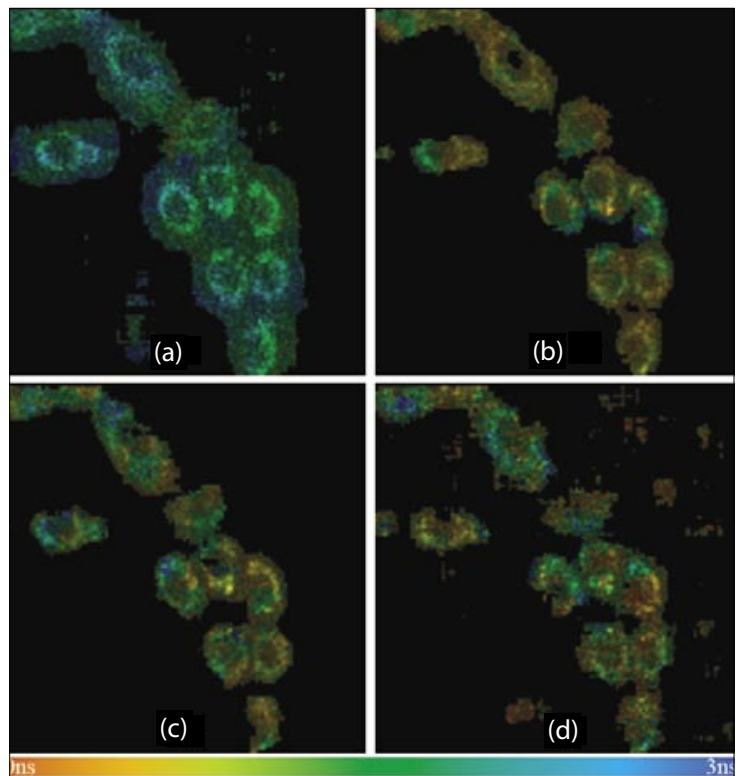

Figure 3. Multiphoton laser-scanning microscopy (MPLSM) spectral/lifetime images of live MCF10 breast cells. Panels depict lifetime images of the cells at the (a) $420 \mathrm{~nm}$, (b) $440 \mathrm{~nm}$, (c) $470 \mathrm{~nm}$, and (d) $510 \mathrm{~nm}$ wavelength components of the emission range. Autofluorescence is from nicotinamide adenine dinucleotide (NADH), and cells were excited at $780 \mathrm{~nm}$. 


\section{Tech Insight}

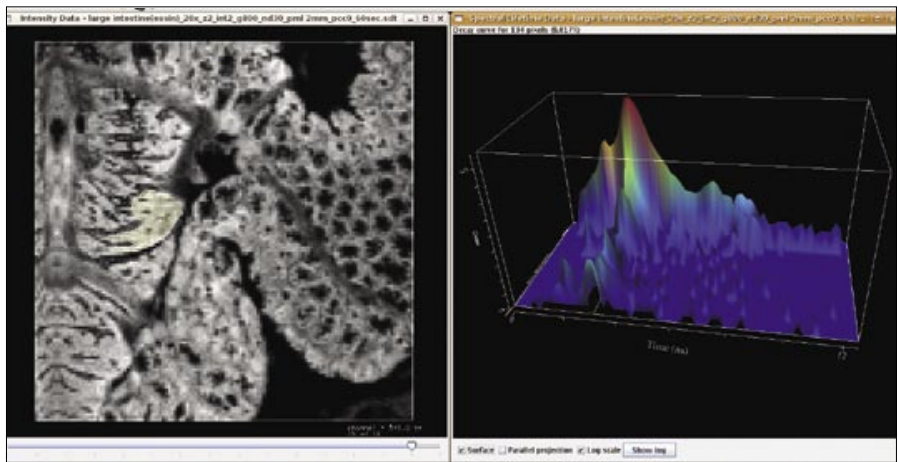

Figure 4. Pilot implementation of spectral lifetime imaging microscopy (SLIM) plotter application for spectral/lifetime visualization. The left panel displays an intensity image at each spectral channel (all lifetime bins summed together to produce an aggregate pixel value). A region of interest has been drawn by hand around pixels of interest, and the right panel shows a summed lifetime curve (along the time axis) at each spectral channel (wavelength axis) for the selected pixels. From the figure, it can be seen that the largest quantity of fluorescence took place across channels 14, 15, and 16 (approximate wavelength range 530-550).

\section{Future Directions}

The SLIM technique we have reported in this manuscript has great potential for cell biology applications. However, the limited photon counting rates of currently available instrumentation reduces the dynamic range of measurements and necessitates the use of long exposure times. This means that for some applications SLIM is impractical, as the photon-counting times needed are too long to capture the biological event in question. For widespread adoption, where scientists would routinely collect spectral and lifetime dimensions much in the same way that temporal and spatial dimensions are collected, great improvements in photon-counting electronics need to be made. New timing electronics have recently become available, so it is only a matter of time before these speed improvements are realized, allowing for shorter photon-counting times.

In addition to spectral and lifetime, fluorescence has another potentially useful property, polarization, which is the measure of the rotational state of the molecule. This property is, for the most part, not used in biology microscopy, but has great potential $(16,46)$. Future systems could detect polarization for several types of studies including metabolic mapping of tumors (17) and measurements of receptor mobility in membranes (46).

Another ongoing challenge with SLIM is data analysis. The collection of simultaneous spectral and lifetime dimensions present practical challenges of how to store these data, due to their large size and complex structure. As well, there is the need for advanced approaches for mapping the data as quickly and effectively as possible. We have developed a software tool for performing analysis of combined spectral/lifetime data (Figure 4). It allows the specification of a freehand region of interest (ROI) to identify pixels of interest, then bins those pixels together to produce an aggregate lifetime decay curve for each spectral channel, plotting the results in an interactive 3-D window. We plan to introduce additional features into the software, such as the ability to bin multiple spectral channels together and export the results for use in other lifetime analysis products, such as the SPCImage program (Becker \& Hickl GmbH, Berlin, Germany) for performing lifetime curve fitting.

\section{Acknowledgments}

This work was supported under National Institutes of Health (NIH) National Institute of Biomedical Imaging and BioEngineering (NIBIB) grant no. R01-EB000184.

\section{References}

1. Schwann, T. 1839. Mikroskopische Untersuchungen über die Übereinstimmung in der Struktur und dem Wachstum der Tiere und Pflanzen. Sander'schen Buchhandlung, Berlin.

2. Scheiden, M. 1838. Beitrfige zur Phytogenesis. Arch. Anat. Physiol. Wiss. Med. 13:137-176.

3. Mazzarello, P. 1999. A unifying concept: the history of cell theory. Nat. Cell Biol. 1:E13-E15.

4. Zhang, S., C. Ma, and M. Chalfie. 2004. Combinatorial marking of cells and organelles with reconstituted fluorescent proteins. Cell 119:137-144.

5. Zaccolo, M., F. De Giorgi, C.Y. Cho, L. Feng, T. Knapp, P.A. Negulescu, S.S. Taylor, R.Y. Tsien, and T. Pozzan. 2000. A genetically encoded, fluorescent indicator for cyclic AMP in living cells. Nat. Cell Biol. 2:25-29.

6. Zaccolo, M. and T. Pozzan. 2000. Imaging signal transduction in living cells with GFP-based probes. IUBMB Life 49:375-379.

7. Zacharias, D.A., G.S. Baird, and R.Y. Tsien. 2000. Recent advances in technology for measuring and manipulating cell signals. Curr. Opin. Neurobiol. 10:416-421.

8. Zacharias, D.A. and R.Y. Tsien. 2006. Molecular biology and mutation of green fluorescent protein. Methods Biochem. Anal. 47:83-120.

9. Tour, O., R.M. Meijer, D.A. Zacharias, S.R. Adams, and R.Y. Tsien. 2003. Genetically targeted chromophore-assisted light inactivation. Nat. Biotechnol. 21:1505-1508.

10. Chalfie, M., Y. Tu, G. Euskirchen, W.W. Ward, and D.C. Prasher. 1994. Green fluorescent protein as a marker for gene expression. Science 263:802-805.

11. Nakai, J., M. Ohkura, and K. Imoto. 2001. A high signal-to-noise $\mathrm{Ca}(2+)$ probe composed of a single green fluorescent protein. Nat. Biotechnol. 19:137-141.

12. Yu, D., G.S. Baird, R.Y. Tsien, and R.L. Davis. 2003. Detection of calcium transients in Drosophila mushroom body neurons with camgaroo reporters. J. Neurosci. 23:64-72.

13. Yu, R. and P.M. Hinkle. 2000. Rapid turnover of calcium in the endoplasmic reticulum during signaling. Studies with cameleon calcium indicators. J. Biol. Chem. 275:23648-23653.

14. Tramier, M., I. Gautier, T. Piolot, S. Ravalet, K. Kemnitz, J. Coppey, C. Durieux, V. Mignotte, and M. Coppey-Moisan. 2002 Picosecond-hetero-FRET microscopy to probe protein-protein interactions in live cells. Biophys. J. 83:3570-3577.

15. Miyawaki, A., J. Llopis, R. Heim, J.M. McCaffery, J.A. Adams, M. Ikura, and R.Y. Tsien. 1997. Fluorescent indicators for Ca2+ based on green fluorescent proteins and calmodulin. Nature 388:882-887. 
16. Gautier, I., M. Tramier, C. Durieux, J. Coppey, R.B. Pansu, J.C. Nicolas, K. Kemnitz, and M. Coppey-Moisan. 2001. HomoFRET microscopy in living cells to measure monomer-dimer transition of GFP-tagged proteins. Biophys. J. 80:3000-3008.

17. Rao, M. and S. Mayor. 2005. Use of Forster's resonance energy transfer microscopy to study lipid rafts. Biochim. Biophys. Acta 1746:221-233.

18. Skala, M.C., J.M. Squirrell, K.M. Vrotsos, J.C. Eickhoff, A. Gendron-Fitzpatrick, K.W. Eliceiri, and N. Ramanujam. 2005. Multiphoton microscopy of endogenous fluorescence differentiates normal, precancerous, and cancerous squamous epithelial tissues. Cancer Res. 65:1180-1186.

19. White, J., J.M. Squirrell, and K.W. Eliceri. 2001. Applying multiphoton imaging to the study of membrane dynamics in living cells. Traffic November.

20. Williams, R.M., W.R. Zipfel, and W.W. Webb. 2001. Multiphoton microscopy in biological research. Curr. Opin. Chem. Biol. 5:603-608.

21. Denk, W., J.H. Strickler, and W.W. Webb. 1990. Two-photon laser scanning fluorescence microscopy. Science 248:73-76.

22. Campagnola, P.J., A.C. Millard, M. Terasaki, P.E. Hoppe, C.J. Malone, W.A. Mohler. 2002. Three-dimensional high-resolution second-harmonic generation imaging of endogenous structural proteins in biological tissues. Biophys. J. 82:493-508.

23. Campagnola, P.J., H.A. Clark, W.A. Mohler, A. Lewis, and L.M. Loew. 2001. Second-harmonic imaging microscopy of living cells. J. Biomed. Opt. 6:277-286.

24. Zoumi, A., A. Yeh, and B.J. Tromberg. 2002. Imaging cells and extracellular matrix in vivo by using second-harmonic generation and two-photon excited fluorescence. Proc. Natl. Acad. Sci. USA 99:11014-11019.

25. Williams, R.M., W.R. Zipfel, and W.W. Webb. 2005. Interpreting second-harmonic generation images of collagen I fibrils. Biophys. J. 88:1377-1386.

26. Bastiaens, P.I. and A. Squire. 1999. Fluorescence lifetime imaging microscopy: spatial resolution of biochemical processes in the cell. Trends Cell Biol. 9:48-52.

27. Pepperkok, R., A. Squire, S. Geley, and P.I. Bastiaens. 1999. Simultaneous detection of multiple green fluorescent proteins in live cells by fluorescence lifetime imaging microscopy. Curr. Biol. 9:269-272.

28. Lakowicz, J.R., H. Szmacinski, K. Nowaczyk, K.W. Berndt, and M. Johnson. 1992. Fluorescence lifetime imaging. Anal. Biochem. 202:316-330.

29. Bird, D.K., L. Yan, K.M. Vrotsos, K.W. Eliceiri, E.M. Vaughan, P.J. Keely, J.G. White, and N. Ramanujam. 2005. Metabolic mapping of MCF10A human breast cells via multiphoton fluorescence lifetime imaging of the coenzyme NADH. Cancer Res. 65:8766-8773.

30. Eliceiri, K.W., C.H. Fan, G.E. Lyons, and J.G. White. 2003. Analysis of histology specimens using lifetime multiphoton microscopy. J. Biomed. Opt. 8:376-380.

31. Parsons, M., J. Monypenny, S.M. Ameer-Beg, T.H. Millard, L.M. Machesky, M. Peter, M.D. Keppler, G. Schiavo, et al. 2005. Spatially distinct binding of Cdc42 to PAK1 and N-WASP in breast carcinoma cells. Mol. Cell. Biol. 25:1680-1695.

32. Peter, M., S.M. Ameer-Beg, M.K. Hughes, M.D. Keppler, S. Prag, M. Marsh, B. Vojnovic, and T. Ng. 2005. MultiphotonFLIM quantification of the EGFP-mRFP1 FRET pair for localization of membrane receptor-kinase interactions. Biophys. J. 88:1224-1237.

33. Mohler, W.A. and J.G. White. 1998. Multiphoton laser scanning microscopy for four-dimensional analysis of $C$. elegans embryonic development. Opt. Express 3:325-331.

34. Ahmed, F., J. Wyckoff, E.Y. Lin, W. Wang, Y. Wang, L. Hennighausen, J. Miyazaki, J. Jones, et al. 2002. GFP expression in the mammary gland for imaging of mammary tumor cells in transgenic mice. Cancer Res. 62:7166-7169.

35. Lakowicz, J.R., H. Szmacinski, K. Nowaczyk, and M.L. Johnson. 1992. Fluorescence lifetime imaging of free and protein-bound NADH. Proc. Natl. Acad. Sci. USA 89:1271-1275.

36. Bird, D.K., K.W. Eliceiri, C.H. Fan, and J.G. White. 2004. Simultaneous two-photon spectral and lifetime fluorescence microscopy. Appl. Opt. 43:5173-5182.

37. Hanley, Q.S., D.J. Ardnt-Jovin, and T.M. Jovin. 2002. Spectrally resolved fluorescence lifetime imaging microscopy. Appl. Spectrosc. 56:155-166.

38. Becker, W., A. Bergmann, E. Haustein, Z. Petrasek, P. Schwille, C. Biskup, L. Kelbauskas, K. Benndorf, et al. 2006. Fluorescence lifetime images and correlation spectra obtained by multidimensional time-correlated single photon counting. Microsc. Res. Tech. 69:186-195.

39. White, J.G., W.B. Amos, and M. Fordham. 1987. An evaluation of confocal versus conventional imaging of biological structures by fluorescence light microscopy. J. Cell Biol. 105:41-48.

40. Amos, W.B. and J.G. White. 2003. How the confocal laser scanning microscope entered biological research. Biol. Cell. 95:335-342

41. Thomas, C., P. DeVries, J. Hardin, and J. White. 1996. Four-dimensional imaging: computer visualization of 3D movements in living specimens. Science 273:603-607.

42. Mohler, W.A. and J.G. White. 1998. Stereo-4-D reconstruc tion and animation from living fluorescent specimens. BioTechniques 24:1006-1012.

43. Squirrell, J.M., D.L. Wokosin, J.G. White, and B.D. Bavister. 1999. Long-term two-photon fluorescence imaging of mammalian embryos without compromising viability. Nat. Biotechnol. 17:763-767.

44. French, T., P.T. So, D.J. Weaver, Jr., T. Coelho-Sampaio, E. Gratton, E.W. Voss, Jr., and J. Carrero. 1997. Two-photon fluorescence lifetime imaging microscopy of macrophage-mediated antigen processing. J. Microsc. 185:339-353.

45. Lin, H.J., P. Herman, and J.R. Lakowicz. 2003. Fluorescence lifetime-resolved $\mathrm{pH}$ imaging of living cells. Cytometry A. 52:77-89.

46. Vishwasrao, H.D., A.A. Heikal, K.A. Kasischke, and W.W.Webb. 2005. Conformational dependence of intracellular NADH on metabolic state revealed by associated fluorescence anisotropy. J. Biol. Chem. 280:25119-25126. 